\title{
Éduquer à l'environnement sans savoir sur la société: le cas des manuels scolaires québécois du primaire en sciences et technologies
}

\section{Johanne Lebrun et Abdelkrim Hasni}

Le texte présente une analyse de 69 situations d'enseignement-apprentissage (SEA) en sciences et technologies intégrant le domaine général de formation "Environnement et consommation». Les SEA analysées proviennent de manuels scolaires québécois du primaire. L'analyse vise à dégager les dynamiques sociospatiales prises en considération lors du traitement des problématiques environnementales. Les résultats démontrent que malgré l'articulation annoncée du domaine général de formation "Environnement et consommation" aux SEA en sciences et technologies, celles-ci demeurent pour l'essentiel disciplinaires et sollicitent fort peu de savoirs en lien avec les dynamiques sociospatiales. Ainsi, les dimensions territoriales, politiques et économiques sont largement évacuées des réflexions environnementales.

\section{Introduction}

L'environnement est une préoccupation sociale grandissante dans la plupart des pays occidentaux. Le réchauffement climatique, la diminution de la biodiversité, le smog, etc. représentent des situations préoccupantes qui rendent de plus en plus nécessaires et urgentes les actions individuelles et collectives aux niveaux local, national et mondial. Les nombreuses initiatives internationales (par exemple: la Conférence des Nations Unies sur l'environnement humain, Stockholm, 1972; le Séminaire international sur l'éducation relative à l'environnement (ÉRE), Belgrade, 1975; la Conférence internationale sur l'ÉRE, Tbilissi, 1977; le Sommet de la Terre, Rio, 1992, etc.) qui ont eu lieu ces dernières décennies reflètent l'importance accordée aux problématiques environnementales. Ces préoccupations ont conduit plusieurs pays à la prise en compte, sous des appellations et des modes de présence divers, de l'ÉRE dans les programmes d'études. La quasi-unanimité qui existe quant à la nécessité de cette éducation s'effrite cependant rapidement lorsqu'il est question de l'orientation à lui donner. La polysémie terminologique associée à ce champ éducatif illustre la diversité de finalités et de contenus accolés à l'ÉRE. À titre d'exemple, Sauvé (2006) identifie 
l'existence de 15 courants distincts dans le champ de l'ÉRE tandis que Meirieu (2001), sur la base de la typologie de Guilbert et Gauthier (1998-1999), évoque quatre justifications divergentes pour l'introduction de l'ÉRE dans le monde scolaire. Le champ québécois de l'ÉRE n'échappe pas à l'équivoque, notamment en ce qui a trait aux finalités éducatives dévolues à cette éducation et à son ancrage dans les disciplines scolaires inscrites au curriculum de l'enseignement primaire.

Dans le contexte de l'enseignement des sciences et technologies, l'ÉRE renvoie non seulement à des savoirs composant la structure disciplinaire, mais aussi à des savoirs véhiculant des enjeux sociaux qui interpellent les apprenants individuellement et dans leur appartenance à la collectivité. Ces savoirs que suscite l'ÉRE dans le cadre de l'enseignement scientifique sont de nature variée: on peut distinguer d'une part les savoirs scientifiques qui appuient la compréhension de problématiques comme la biodiversité, le réchauffement climatique, l'extinction des espèces, etc., et d'autre part les savoirs disciplinaires autres que les savoirs scientifiques. Il s'agit notamment des savoirs en provenance des disciplines des sciences humaines et sociales. C'est le cas par exemple lorsqu'il s'agit de comprendre la répartition animale ou végétale d'un écosystème dans une perspective qui tient compte des considérations économiques, sociales et culturelles des populations humaines; enfin, les savoirs non scientifiques renvoient aux «bonnes» actions citoyennes qui ne s'appuient pas nécessairement sur des apprentissages disciplinaires particuliers, comme le recyclage à l'école ou la participation avec le groupe classe à la plantation d'arbres dans une zone défrichée.

Dans le cadre restreint de ce texte, nous nous intéressons à la question de l'articulation des savoirs d'autres disciplines à l'ÉRE. De manière plus spécifique, il s'agit ici d'analyser l'intégration de savoirs provenant du programme d'enseignement d'histoire et de géographie à l'ÉRE (Ministère de l'Éducation, 2001). Plusieurs contenus de ce programme d'enseignement, centré sur l'étude des dynamiques sociospatiales, sur l'évolution et la diversité des organisations sociales et territoriales, sont susceptibles d'éclairer les problématiques environnementales. En effet, le processus d'anthropisation ou d'aménagement et d'exploitation du cadre de vie naturel des sociétés ainsi que les raisons expliquant la diversité des processus et des résultats de cette anthropisation sont désormais au cœur des apprentissages visés en histoire et géographie.

Le texte vise à circonscrire les savoirs historiques et géographiques pris en considération dans les manuels scolaires québécois du primaire en sciences et technologies lors du traitement du domaine général de formation (DGF) «Environnement et consommation». L'analyse a été effectuée dans trois manuels scolaires ${ }^{1}$ dits interdisciplinaires ou transdisciplinaires. Ceux-ci intègrent l'enseignement des sciences et technologies, de l'histoire-géographie, du français et dans certains cas des arts. Ces manuels présentent une structuration potentiellement plus propice à l'intégration des savoirs historiques et géographiques au traitement du DGF «Environnement et consommation» dans les situations d'enseignement-apprentissage (SEA) en sciences et technologies. 
Il s'agit d'une étude per se du manuel pour reprendre les propos de Luke, de Castell et Luke (1989) qui distinguent entre deux principales orientations dans la recherche sur le manuel scolaire: celles qui traitent du manuel des sciences comme objet d'étude "per se» et celles qui l'abordent comme outil, «in use». En tant qu'objet, le manuel véhicule une conception du savoir, une manière de considérer l'enseignement et l'apprentissage, qu'il est important d'analyser (Hasni, Samson, Moresoli \& Owen, 2009), d'autant plus que le Ministère de l'Éducation du Québec (MEQ) attribue un rôle prépondérant aux manuels scolaires dans l'actualisation de la réforme curriculaire centrée sur l'approche par compétences amorcée en 2001. La Commission du matériel didactique (2002) attribue huit fonctions aux manuels scolaires: médiation entre le programme et les enseignants; soutien à l'enseignement; support à l'apprentissage; référent pour l'élève et ses aidants (par exemple les parents); rehaussement culturel; promotion de valeurs sociétales; garantie de la gratuité scolaire; supervision pédagogique. Les critères d'approbation ont été aussi renouvelés afin de répondre à ces nouvelles fonctions (MEQ, 2004). Cette place accordée aux manuels scolaires dans la mise en œuvre du curriculum exige qu'ils soient sérieusement considérés dans les recherches en éducation, comme en témoignent les nombreuses manifestations scientifiques internationales organisées au cours des dernières années (par exemple le Séminaire international sur les manuels scolaires organisé en 2006 au Chili, suite à une recommandation de l'OCDE, les récents congrès internationaux organisés sur le sujet par le Laboratoire sur les manuels scolaires en 2006 et par l'International Organisation for Science and Technology Education en 2007).

Suite à l'identification des zones d'ombre marquant l'inscription curriculaire du DGF «Environnement et consommation» dans le programme québécois pour l'enseignement primaire, le texte présente successivement les paramètres conceptuels et méthodologiques qui ont présidé à l'analyse des manuels scolaires puis les résultats de cette analyse. Ceux-ci sont ensuite discutés de manière à mettre en exergue les savoirs historiques et géographiques qui sont pris en considération dans l'éducation à l'environnement et à la consommation ainsi que la fonction de ces savoirs dans le traitement de ce champ éducatif.

\section{L'éducation à L'environnement dans le contexte éducatif québécois: entre la distanciation et l'adhésion, entre la disciplinarité et l'interdisciplinarité}

Le programme de formation du primaire (Gouvernement du Québec, 2001) comporte trois composantes interreliées: les domaines généraux de formation, les compétences disciplinaires et les compétences transversales. L’enseignement disciplinaire doit maintenant concourir au développement de la capacité de l'élève à choisir, à mobiliser, à combiner des ressources pour répondre à des situ- 
ations complexes et singulières. Le développement des compétences disciplinaires nécessite ainsi la mise en ouvre de situations d'apprentissages ancrées dans la réalité sociale et individuelle des élèves. Les DGF participent à cet effort de contextualisation des savoirs. Ils regroupent «un ensemble de grandes questions que les jeunes doivent affronter. Ils rapprochent les savoirs disciplinaires des préoccupations quotidiennes de l'élève et lui donnent plus de prise sur la réalité» (Gouvernement du Québec, 2001, p. 42).

L'ÉRE trouve son point d'ancrage curriculaire dans le DGF «Environnement et consommation». Comme les autres DGF («Santé et bien-être», "Orientation et entrepreneuriat», "Environnement et consommation», «Médias» et "Vivre ensemble et citoyenneté»), il doit «se développer par et à travers les autres apprentissages tout en leur permettant de s'inscrire dans des problématiques proches de la vie (Gouvernement du Québec, 2001, p. 42). L'encadré 1 présente l'intention éducative et les axes de développement associés au domaine «Environnement et consommation».

Encadré 1: Intention éducative et axes de développement associés au domaine «Environnement et consommation»

\section{Intention éducative}

Amener l'élève à entretenir un rapport dynamique avec son milieu, tout en gardant une distance critique à l'égard de l'exploitation de l'environnement, du développement technologique et des biens de consommation (p. 47).

\section{Axes de développement}

Présence à son milieu: sensibilité à l'environnement naturel et humain; compréhension de certaines caractéristiques et de phénomènes de son milieu; représentation spatiale (orientation, localisation, tracé, plan, etc.); identification des liens entre les éléments propres à un milieu local ou régional, à une saison; reconnaissance de l'interdépendance entre l'environnement et l'activité humaine. Faire le partage entre ses besoins réels et ses désirs.

Construction d'un environnement viable dans une perspective de développement durable: lien entre la satisfaction des besoins des membres d'une collectivité et le territoire sur lequel ils évoluent; utilisation rationnelle des ressources en fonction des besoins de tous les êtres vivants; habitudes et attitudes visant la protection, la conservation et l'amélioration de l'environnement (actions personnelles et collectives de récupération, de recyclage et de réutilisation); incidences de la science et de la technologie et questionnement sur les usages qui en sont faits; respect du patrimoine.

Stratégies de consommation et d'utilisation responsable de biens et de services: distinction entre désirs et besoins; sources d'influence liées à la consommation (médias, famille, amis, groupes, etc.); étapes d'une stratégie de consommation efficace (établir les objectifs, recueillir de l'information, déterminer ses dépenses et les équilibrer en fonction de son budget).

Conscience des aspects sociaux, économiques et éthiques du monde de la consommation: interdépendance des personnes, des peuples et de leurs réalisations; provenance des divers produits consommés; conséquences de la mondialisation sur les cultures, les modes de vie et la répartition de la richesse; conditions de travail des producteurs de biens et de services et moyens de les améliorer; choix de consommation dans le respect de la qualité de vie personnelle et collective.

Source: Ministère de l'Éducation (2001). Programme de formation de l'école québécoise au préscolaire et au primaire, pp. 46-47.

La première équivoque en regard de ce champ éducatif se situe sur le plan des finalités. Dans les programmes, les axes de développement se situent tantôt sur le plan de l'adhésion à des valeurs prédéterminées (par exemple, choix de consom- 
mation dans le respect de la qualité de vie personnelle et collective), tantôt sur le plan de l'analyse réflexive et critique (par exemple, questionnements sur les usages de la science et de la technologie). Le Conseil supérieur de l'éducation (CSE) (2007), quant à lui, place les DGF en lien avec les valeurs. Pour le CSE, ils sont «imprégnés d'une disposition à proposer des valeurs, disposition dont on ne trouve pas forcément l'équivalent dans l'étude des disciplines spécialisées» (p. 9).

La seconde équivoque se situe sur le plan de la spécificité des contenus de certains axes du DGF «Environnement et consommation» par rapport au champ disciplinaire des sciences et technologies. Des éléments tels les interactions entre l'homme et son milieu, les phénomènes de son milieu, la consommation et la conservation de l'énergie, les techniques de recyclage et de compostage, les transports, etc. chevauchent les contenus et les repères culturels du programme d'études en sciences et technologies. Les repères culturels sont définis par le MEQ (2001) comme des informations complémentaires qui «permettent de mettre en perspective, d'enrichir, de personnaliser, de nuancer et de mieux intégrer les compétences» (p. 156). Le MEQ (2001) identifie sept catégories de repères culturels: les liens entre les sciences et technologies et les autres champs de l'activité humaine, le contexte historique entourant le développement scientifique et technologique, les grands scientifiques, les valeurs fondamentales de la science, les enjeux éthiques de la science, les impacts de la science et de la technologie ainsi que ses limites.

De tels chevauchements sont susceptibles de contribuer à la dilution de l'ÉRE. Si celle-ci est considérée comme étant sous-jacente, voire naturellement incluse, dans les SEA en sciences et technologies, son intégration pourra se limiter par exemple à joindre un questionnement sur sa consommation d'eau à une SEA portant sur les diverses utilisations de l'eau. Des données de recherche laissent entrevoir que cette conception est éventuellement présente chez certains acteurs du milieu éducatif. Une enquête par questionnaire menée auprès de 841 étudiants en formation initiale à l'enseignement préscolaire et primaire provenant de quatre universités francophones du Québec met en relief que les DGF semblent perçus comme des prolongements des disciplines inscrites au cursus scolaire (Lebrun, Lenoir, Araújo-Oliveira, Morin \& McConnel, sous presse). Ainsi, le DGF «Environnement et consommation» est associé dans une proportion de 80,2\% à l'enseignement des sciences et technologies. Une telle perspective peut conduire à considérer les DGF comme des thématiques directement liées aux objets d'études des disciplines scolaires et dont le traitement ne requiert aucune intervention spécifique.

Force est de reconnaître que l'articulation des DGF aux SEA demeure problématique. En 2006, dans un rapport dressant le bilan de l'application du nouveau programme de formation à l'enseignement primaire, la Table de pilotage du renouveau pédagogique $(2006)^{2}$ souligne la difficulté des enseignants à prendre en considération les domaines généraux de formation. L'une des recommandations de ce rapport est de demander un avis au CSE sur la pertinence et l'appli- 
cabilité des domaines généraux de formation. Dans son avis publié un an plus tard, le CSE (2007) affirme que la pertinence et l'applicabilité des domaines généraux ne fait aucun doute, mais que l'absence de repères dans le programme de formation pour leur intégration dans les différentes disciplines constitue un obstacle important. Leur articulation aux SEA disciplinaires pourrait être aussi problématique dans les manuels scolaires. Les critères d'évaluation édictés par le Ministère de l'Éducation, du Loisir et du Sport (MELS) pour l'approbation des manuels scolaires, se limitent à exiger que la majorité des situations d'enseignement-apprentissage présentées couvrent un ou des DGF, ainsi que leurs axes de développement (MELS, 2004) sans que ne soit soupesé le traitement effectif de ces domaines dans les SEA.

\section{Éléments du cadre conceptuel}

Quels que soient son intitulé et son mode d'inscription curriculaire, l'ÉRE renvoie inévitablement à des enjeux socioéconomiques et sociopolitiques. À l'instar de Lange et Martinand (2010), nous considérons ce champ éducatif, d'une part, comme un ensemble de problématiques nécessitant des choix collectifs de stratégies pour l'avenir et, d'autre part, comme une éducation qui dépasse largement l'enseignement des sciences de l'environnement ou de l'écologie en raison de la nature socioéconomique et sociopolitique des enjeux sous-jacents aux problématiques environnementales. Exclure les dimensions politiques et économiques de l'analyse des problématiques environnementales reviendrait à postuler implicitement la primauté des responsabilités et des actions individuelles. Par ailleurs, comme le souligne Simonneaux (2007) au sujet des questions socialement vives, elles «exigent pour leur résolution plus qu'une solution scientifique, c'est-à-dire la prise en considération des implications sociales, des idéologies et des valeurs qui accompagnent les décisions». L'ÉRE nécessite ainsi des fréquents allers et retours entre l'individuel et le social, entre le politique et l'économique et les valeurs, entre une perspective interdisciplinaire pour l'appréhension de la complexité des problématiques et le disciplinaire pour l'analyse de certains aspects spécifiques.

Lange et Martinand (2010), reprenant les travaux de Sadji et Lange (2007), identifient trois postures quant aux pratiques d'enseignement en lien avec l'éducation au développement durable: la posture de l'implicite où cette éducation est considérée comme naturellement incluse dans l'enseignement disciplinaire; la posture de la greffe où l'enseignement disciplinaire se prolonge par un questionnement ou une ouverture sur des problématiques environnementales et la posture du porteur de projet où la problématique environnementale est centrale. L'importance accordée aux savoirs historiques et géographiques ainsi que la nature des savoirs sollicités sont susceptibles de varier fortement selon les postures adoptées. 
Dans la posture de l'implicite, tout se passe comme si l'enseignement des savoirs écologiques est en lui-même porteur d'une ÉRE. Essentiellement confiné aux contenus disciplinaires de sciences et technologies, cette posture est peu susceptible d'intégrer ou de s'alimenter aux savoirs historiques et géographiques.

Dans une posture de la greffe, l'ÉRE est considérée comme un ensemble de thématiques qui se superposent aux apprentissages disciplinaires. En ce sens, la discipline scolaire est première. Elle vient soit mettre en lumière de manière essentiellement descriptive et factuelle une problématique environnementale, soit circonscrire un phénomène, une thématique, qui pourra par la suite faire l'objet d'une réflexion plus spécifique en lien avec le DGF. Les savoirs historiques et géographiques seront alors convoqués pour illustrer, par exemple, l'évolution des problèmes environnementaux ou encore pour localiser les phénomènes étudiés. À prédominante historique, les savoirs convoqués seront de nature déclarative et factuelle.

Dans une posture de projet, l'ÉRE est porteuse de problématiques. Ces problématiques environnementales deviennent alors des éléments structurants pour les SEA. Dans ce cas de figure, les sciences humaines et sociales avec leur angle d'analyse, leur trame conceptuelle et leur mode de raisonnement seront sollicitées pour scruter les enjeux de la problématique environnementale. Ce sont ainsi à la fois les concepts géographiques (par exemple, distribution, différenciation, systèmes de production, flux de marchandises, échelles des phénomènes, urbanisation, etc.), politiques (régime politique, pouvoir, classe sociale, justice, etc.), économiques (système de production, commerce, échanges, niveau de développement, consommation, etc.), culturel (religion, patrimoine, croyances, etc.) et historique (changements, périodisation, évolution, etc.) et les modes de raisonnement géographique et historique ainsi que les supports qui y sont associés (cartes, chorèmes, etc.) qui pourront être sollicités dans l'analyse des problématiques environnementales.

Bien que tracées rapidement et à grands traits, ces trois configurations guident l'analyse de la prise en considération des savoirs sociaux dans l'ÉRE. Il importe de préciser que l'esquisse présentée se veut uniquement une structure de référence pour situer sur un large spectre la ou les perspectives de prise en considération qui se dégagent des manuels scolaires.

\section{Aspects méthodologiques}

Dans la mesure où l'ÉRE est prioritairement associée à l'enseignement des sciences et technologies, notre analyse des manuels scolaires a porté sur les SEA consacrées à cette discipline et auxquelles étaient explicitement annoncée l'intégration de ce DGF. L'analyse s'est effectuée dans des manuels scolaires dits interdisciplinaires ou transdisciplinaires. Bien qu'ils couvrent un ensemble de disciplines scolaires, ces manuels scolaires distinguent nettement l'enseignement 
dans chacune des disciplines. Ainsi, on y retrouve des SEA en sciences et technologies, en histoire-géographie, en français, etc. Toutefois, les SEA en sciences et technologies, par exemple, poursuivent également d'autres apprentissages disciplinaires. Les manuels analysés devaient également être approuvés par le MELS. Trois ensembles répondent à ce critère. Le tableau 1 présente les caractéristiques de ces ensembles didactiques.

\section{Tableau 1: Caractéristiques du corpus d'analyse}

\begin{tabular}{|l|c|c|}
\hline \multicolumn{1}{|c|}{$\begin{array}{c}\text { Références (auteurs, titre, éditeur, } \\
\text { année de production) }\end{array}$} & Cycle (1) & \begin{tabular}{c} 
Disciplines scolaires intégrées \\
\hline $\begin{array}{l}\text { Létourneau, G. et Trudeau, S. (2003). } \\
\text { Mes chantiers. Montréal: Graficor. }\end{array}$
\end{tabular} \\
\hline $\begin{array}{l}\text { Frapet, J., Péladeau, I. et Saint-Pierre, I. } \\
\text { (2003). Ankor. Montréal: Modulo }\end{array}$ & 2 & $\begin{array}{c}\text { Français, sciences et technologies, histoire, géo- } \\
\text { graphie, arts plastiques, art dramatique }\end{array}$ \\
\hline $\begin{array}{l}\text { Lord, F., Lytwynuk, D., Morrissette, I. } \\
\text { et Péladeau, J. (2004). Cyclades. Mont- } \\
\text { réal: Modulo. }\end{array}$ & 3 & $\begin{array}{c}\text { Français, sciences et technologies, histoire, géo- } \\
\text { graphie, arts plastiques, art dramatique }\end{array}$ \\
\hline
\end{tabular}

(1) Au Québec, l'enseignement primaire est divisé en trois cycles. Le premier cycle du primaire couvre la 1ère et la 2 e année du primaire (élèves de 6 ans et de 7 ans). Le deuxième cycle du primaire englobe la $3 e$ et la $4 \mathrm{e}$ année (élèves de 8 et 9 ans) alors que le troisième cycle englobe la $5 \mathrm{e}$ et la 6e année (élèves de 10 et 11 ans).

Au Québec, les manuels sont composés d'un ou plusieurs guides d'enseignement et d'un ou plusieurs manuels de l'élève. Les guides d'enseignement contiennent une partie théorique qui présente les orientations, les fondements, les approches pédagogiques, évaluatives, etc. en reprenant largement le discours ministériel, et un versant opératoire qui contient les activités des manuels de l'élève et des précisions quant à leur conduite. Ce sont donc les guides qui ont fait l'objet de l'analyse puisqu'ils permettent d'accéder à la fois aux orientations fournies à l'enseignant et aux activités du manuel de l'élève. Le premier niveau d'analyse touche le discours introductif explicite du guide de l'enseignant au sujet des domaines généraux de formation. Les passages référant explicitement au DGF et à leur intégration aux enseignements disciplinaires ont été relevés. Ces éléments de discours servent tout au plus à contextualiser le traitement des DGF dans les SEA. Le deuxième niveau d'analyse concerne les visées d'apprentissage et les tâches associées aux SEA en sciences et technologies intégrant le domaine "Environnement et consommation». Pour les deux niveaux, la procédure d'analyse retenue repose sur une grille d'analyse thématique mixte où une partie des catégories analytiques découle de la structure conceptuelle de référence alors qu'une autre partie émerge du matériel analysé (Boyatzis, 1998; Landry, 1998).

Pour les visées d'apprentissage, les éléments analysés sont variables d'un ensemble didactique à l'autre. Dans l'ensemble didactique Mes chantiers, nous avons utilisé les questions présentées aux élèves en amorce de chacune des SEA en sciences et technologies intégrant le DGF environnement et consommation. 
Les élèves doivent trouver les réponses à ces questions au fil de l'activité. Pour Ankor et Cyclades nous nous sommes appuyés sur le résumé de chaque SEA dans lequel sont identifiés les apprentissages que les élèves devront réaliser. L'analyse de ces résumés ou des questions soumises aux élèves permet de dégager l'intégration annoncée ou non d'éléments en lien avec l'ÉRE. Il s'agit ici de vérifier si l'annonce officielle de l'intégration de l'ÉRE à la SEA se traduit réellement dans les visées d'apprentissage. Chacune des tâches composant les SEA a ensuite été analysée. Cette analyse visait à identifier les savoirs historiques et géographiques réellement mobilisés dans les SEA ainsi que la nature de ces derniers.

Le tableau 2 présente la grille d'analyse. C'est à partir du croisement de l'ensemble des données que la prise en considération des savoirs historiques et géographiques est interprétée.

\section{Tableau 2: Grille d'analyse}

1. Analyse du discours introductif

a. Discours explicite sur l'articulation des domaines généraux de formation aux SEA

b. Discours explicite sur les intentions éducatives en lien avec les SEA en sciences et technologies intégrant l'ÉRE

2. Analyse des visées d'apprentissage dans les SEA en sciences et technologies intégrant officiellement l'ÉRE

a. Présence ou non d'apprentissages explicitement en lien avec l'ÉRE

3. Analyse des tâches proposées aux élèves dans les SEA en sciences et technologies intégrant officiellement l'ÉRE

a. Les savoirs historiques et géographiques sollicités

i. Dimension politique (régime politique, droits et devoirs, classe sociale, services sociaux, justice, etc.)

ii. Dimension économique (système de production, commerce, échanges, niveau de développement, consommation, etc.)

iii. Dimension historique (changements, périodisation, évolution, etc.)

iv. Dimension spatiale (localisation, distribution, différenciation, systèmes de production, flux de marchandises, échelles des phénomènes, etc.)

v. Dimension culturelle (religion, patrimoine, croyances, etc.)

vi. Autre (spécifier)

vii. Aucune

b. La nature des savoirs historiques et géographiques sollicités

i. Savoir factuel (dates, événements, personnages, lieux, etc.)

ii. Savoir conceptuel (organisation politique, système de production, etc.)

iii. Autre (spécifier)

Le tableau 3 présente la fréquence d'association des différents DGF aux SEA en sciences et technologies dans chacun des ensembles didactiques. Les SEA analysées sont celles qui articulent le DGF «Environnement et consommation». Au total, ce sont 69 SEA qui ont été analysées, ce qui correspond respectivement à $74 \%, 81 \%$ et $90 \%$ de la totalité des SEA en sciences et technologies contenues dans Ankor, dans Mes Chantiers et dans Cyclades. 
Tableau 3: Fréquence d'association des DGF aux SEA en sciences et technologies dans les trois ensembles didactiques

\begin{tabular}{|l|c|c|c|}
\hline & Mes chantiers & Ankor $^{\mathbf{2}}$ & Cyclades $^{3}$ \\
\hline Environnement et consommation & 13 & 36 & 20 \\
\hline Vivre ensemble et citoyenneté & 0 & 3 & 3 \\
\hline Médias & 0 & 2 & 7 \\
\hline Santé et bien-être & 3 & 5 & 8 \\
\hline Orientation et entrepreneuriat & 0 & 18 & 27 \\
\hline Total des SEA en sciences et technologies & 16 & 40 & 7 \\
\hline
\end{tabular}

(1) L'ensemble didactique Mes chantiers associe un seul DGF à chacune des SEA.

(2) L'ensemble didactique Ankor associe de un à quatre DGF à chacune des SEA.

(3) L'ensemble didactique Cyclades associe de un à quatre DGF à chacune des SEA.

\section{Résultats}

Les résultats sont présentés pour chacun des ensembles didactiques dans l'ordre suivant: d'abord les extraits du discours explicite en lien avec les domaines généraux de formation, puis les résultats des analyses des SEA.

\section{L'ensemble didactique Mes chantiers}

Mes Chantiers est un ensemble didactique qui se dit interdisciplinaire. Les données recueillies montrent qu'au-delà de la reprise d'éléments du discours ministériel, le guide d'enseignement Mes Chantiers apporte peu de précisions quant à l'articulation privilégiée entre les DGF et les SEA. On peut y lire qu'à "partir de grands thèmes liés aux domaines généraux de formation, on aborde les contenus au programme en science et technologie, en géographie, histoire et éducation à la citoyenneté, ainsi qu'en français» (Mes chantiers, guide général, 2003, p. 4). On précise que:

Dans la collection Mes chantiers, au moins un domaine général de formation a été ciblé pour chacun des modules à la lumière des sujets traités. Pour chacune des thématiques, ce domaine constitue le point d'ancrage avec la réalité. Il en assure la signifiance (Mes Chantiers, guide général, 2003, p. 15).

Le discours est tout aussi peu explicite quant aux intentions éducatives sous-jacentes à l'articulation de l'ÉRE avec l'enseignement des sciences et technologies. Des intentions éducatives générales sont énoncées pour l'ensemble des SEA composant un module. Chaque module comporte entre quatre et cinq SEA en sciences et technologies. Pour le module Air et eau, le guide d'enseignement spécifie les intentions éducatives suivantes:

Les élèves reconnaissent que l'étude de la matière, de l'air et de l'eau leur permet de se sensibiliser à l'environnement et à la disponibilité des ressources. Ils comprennent que la Terre constitue la réserve de ce [dont] tous les habitants ont besoin 
pour survivre et qu'il est nécessaire d'en conserver les ressources (Mes Chantiers, guide A, module 8, 2003, p. 12).

Pour ce qui est du Monde vivant,

Les élèves reconnaissent d'abord qu'ils font partie du monde vivant. Ensuite, ils comprennent que l'étude du vivant leur rappellera certains des besoins fondamentaux et des façons de les satisfaire en plus de les sensibiliser dans une large perspective à leur environnement (Mes Chantiers, guide B, module 11, 2003, p. 12).

Enfin, pour le module intitulé Météo et chrono,

Les élèves se sensibilisent au domaine général de formation: environnement et consommation. Ils reconnaissent que l'étude du temps qu'il fait et du temps qui passe offre une belle occasion d'approfondir leur connaissance des divers éléments de leur milieu. Elle les aide aussi à reconnaître que l'essor de la science et de la technologie leur permet de raffiner leur regard sur le monde (Mes Chantiers, guide B, module 17, 2003, p. 12).

Ces intentions sous-tendent que l'ÉRE repose sur le développement d'une connaissance élémentaire de son environnement naturel.

\section{Les séquences d'enseignement-apprentissage}

Dans Mes chantiers, 13 SEA en sciences et technologies sont associées au DGF «Environnement et consommation». De ce nombre, seule une SEA sur 13 comporte une visée explicite d'apprentissage en lien avec le DGF «Environnement et consommation». Intitulée $\vec{A}$ l'eau, cette SEA s'amorce par les questions suivantes: "Que sais-tu des réserves d'eau de la planète? Sous quels états l'eau est-elle disponible sur Terre? Que penses-tu de ta consommation d'eau?» (Mes Chantiers, guide A, module 8,2003 , p. 53). Située sur le plan individuel et familial, cette SEA n'intègre aucune tâche comportant des éléments de réflexion à caractère communautaire. En effet, au sein de cette SEA, les élèves sont d'abord invités à exprimer leurs connaissances préalables sur les réserves d'eau potable mondiale et sur leur consommation personnelle d'eau. Ils lisent ensuite un texte informatif sur l'importance, l'utilisation et la répartition de l'eau, puis procèdent au calcul de la consommation d'eau de leur famille. La SEA se termine par un échange sur les moyens de réduire leur consommation d'eau.

Toutefois quatre SEA ne comportant aucune visée explicite d'apprentissage en lien avec l'ÉRE réferent à des savoirs historiques et géographiques. Ainsi, une SEA portant sur la flottaison comporte la lecture d'un texte informatif sur le naufrage du Titanic. Une autre intitulée Voler du rêve à la réalité propose la lecture d'un texte informatif sur l'évolution des engins volants et invite les élèves à situer sur une ligne du temps les étapes du progrès de l'aviation. En guise de synthèse, le guide d'enseignement suggère d'effectuer un échange sur les impacts sociaux positifs du développement de l'aviation. Le grand verglas de 1998, une ac- 
tivité visant l'étude des phénomènes météorologiques, comporte la lecture de six courts témoignages de gens ayant subi les contrecoups du verglas de 1998 au Québec. Ces témoignages renvoient notamment aux activités économiques et aux services publics. Enfin, une SEA propose la lecture d'un texte informatif sur le vent et son exploitation énergétique par certaines communautés humaines. De nature factuelle, ces savoirs correspondent pour l'essentiel aux repères culturels suggérés par le MELS. Cet ensemble didactique semble donc s'inscrire dans la posture de l'implicite ou dans une perspective strictement disciplinaire au sein de laquelle l'ÉRE serait naturellement incluse dans l'enseignement des sciences et technologies.

\section{L'ensemble didactique Ankor}

Dans son discours introductif, le guide d'enseignement Ankor se limite à énoncer, sans en spécifier les modalités générales ni celles spécifiques à l'ÉRE, que:

dans notre matériel transdisciplinaire, le développement des compétences disciplinaires et transversales sinscrit dans un ensemble de problématiques que les jeunes d'aujourd'hui doivent affronter. Ce sont les domaines généraux de formation. [...] La nature particulière d'un projet donné mettra l'accent sur certains de ces domaines (Ankor, guide AB, 2003, p. 1.11).

Le domaine «Environnement et consommation» est ainsi annoncé comme faisant partie des apprentissages ciblés, mais sans qu'aucune indication ne soit donnée quant à la spécificité de ces apprentissages.

\section{Les séquences d'enseignement-apprentissage}

Dans cet ensemble, on compte de un à quatre DGF associés à chacune des SEA en sciences et technologies. D'emblée, ce nombre important soulève des interrogations quant à l'exploitation effective de ces DGF. Par ailleurs, seules cinq SEA sur 36 comportent des visées d'apprentissage intégrant explicitement des éléments en lien avec l'ÉRE, alors que dix SEA intègrent des savoirs historiques et géographiques. Pour les autres, on se limite à l'énonciation du DGF.

Lorsque les SEA intègrent explicitement des apprentissages en lien avec l'ÉRE, cette éducation se situe dans une posture de la greffe, en ce sens que les SEA en sciences et technologies trouvent leur prolongement dans un questionnement renvoyant à des problématiques environnementales ou liées à la consommation. Par exemple, dans une SEA où les élèves sont conviés à composer un rap de la solidarité, le guide d'enseignement précise que:

La lecture du texte Nous les omnivores amènera les élèves à prendre conscience du rôle prépondérant que joue l'alimentation dans le maintien de la santé. Ils comprendront également qu'il y a un déséquilibre flagrant à cet égard dans le monde: tandis que certains profitent d'une alimentation trop riche, d'autres souffrent de malnutrition. On invitera les élèves à chercher des solutions à ce problème et à composer un rap sur ce thème (Ankor, guide AB, 2003, p. 414). 
Dans une SEA portant sur les animaux dans leur habitat, on peut lire: Les élèves consolideront la notion d'habitat et d'écosystème. L'ensemble des lectures, des recherches et des réflexions déjà entreprises devraient leur permettre de comprendre assez facilement le contenu des deux textes à l'étude. Ils réfléchiront à l'impact de la présence humaine sur l'environnement, et principalement sur la vie animale (Ankor, guide CD, 2003, p. 30).

Dans une activité intitulée Pleins feux sur l'expérimentation, le guide d'enseignement précise que «L'animation du laboratoire commence par la réalisation d'expériences en lien avec l'énergie. [...] Les élèves pourront alors réfléchir à l'importance d'économiser l'énergie et de la consommer dans une perspective de développement durable» (Ankor, guide CD, 2003, p. 345). L'activité Deux siècles d'invention propose aux élèves de monter un kiosque regroupant, entre autres, une collection d'objets techniques qui ont une grande importance dans la vie quotidienne des élèves de la classe.

On situera ces objets sur une ligne du temps, on étudiera leur fonctionnement et on les présentera au salon des inventions, cette situation leur permettra de découvrir les usages variés d'un objet, de retracer son évolution dans l'histoire. Ce sera l'occasion de réfléchir à l'influence de certains objets sur notre façon de vivre et aux conséquences qui sont liées à leur usage (Ankor, guide AB, 2003, p. 552).

Pour l'activité Évolution d'une technologie: une sale histoire, le guide d'enseignement indique:

Les élèves se pencheront d'abord sur une technologie simple et pourtant fondamentale dans notre société: les toilettes à chasse d'eau. Ils étudieront leur fonctionnement en tant qu'objet technique, puis ils liront une brève histoire de nos installations sanitaires et du traitement des eaux usées. Ils seront ainsi amenés à réfléchir aux impacts de l'urbanisation sur la santé (Ankor, guide AB, 2003, p. 568).

Ainsi, lorsque les élèves sont amenés à réfléchir à des dimensions en lien avec l'ÉRE, ils le font en aval des apprentissages en sciences et technologies en s'appuyant sur des savoirs historiques de nature essentiellement factuelle.

Ce ne sont toutefois pas nécessairement les SEA qui annoncent qu'elles poursuivent des apprentissages en lien avec l'ÉRE qui s'appuient le plus sur ces savoirs. Parmi cinq SEA annonçant explicitement viser des apprentissages en lien avec ce DGF, quatre intègrent des savoirs historiques et géographiques à titre de repères culturels, tandis que six SEA ne présentant pas d'apprentissages explicites en lien avec le DGF intègrent également des savoirs historiques. La poursuite d'apprentissages en lien avec l'ÉRE ne s'accompagne donc pas d'une convocation plus importante de ces savoirs. En fait, dans l'ensemble des SEA, les savoirs convoqués renvoient à des repères culturels surtout historiques (par exemple, lecture d'un texte sur les grandes inventions de l'humanité, lecture d'un texte sur Galilée, etc.). 


\section{L'ensemble didactique Cyclades}

Cyclades propose une démarche par projets thématiques dans une perspective transdisciplinaire. Pour chaque thème à l'étude, on trouvera donc un projet intégrateur qui permettra de développer des compétences en touchant les diverses disciplines qui seront mises à contribution pour la réussite du projet (Cyclades, guide $A B, 2004$, p. 5).

À l'instar de l'ensemble didactique précédent, Cyclades ne fournit aucune précision quant aux intentions éducatives présidant à l'articulation du DGF «Environnement et consommation» aux SEA. L'ÉRE est ainsi annoncée comme faisant partie des apprentissages ciblés, mais sans quaucune indication ne soit donnée quant aux apprentissages qui devraient être réalisés.

\section{Les séquences d'enseignement-apprentissage}

Il est à noter que cet ensemble associe également entre un et quatre DGF à chaque SEA, ce qui laisse présager une exploitation superficielle des DGF. En fait, le lien entre les apprentissages visés et le DGF est explicitement présenté dans une seule des 20 SEA. Il renvoie à l'influence de la publicité sur les consommateurs. Dans le cadre de l'amorce de cette SEA intitulée Une consommation nouvelle et améliorée!, les élèves sont invités à échanger, entre autres, sur l'influence de la publicité sur les consommateurs. Il leur est ensuite proposé de réaliser un sondage auprès de 30 consommateurs adultes au regard de deux ou trois marques de produits. Ils vérifient subséquemment leurs impressions et celles des sujets interviewés à l'aide d'un dispositif expérimental. Lors de la phase d'intégration, les élèves doivent questionner les techniques publicitaires et la concordance entre les qualités annoncées et les qualités réelles du produit. Suite à la lecture de la fable Le rat des villes et le rat des champs, ils sont invités à discuter du problème de surconsommation mondiale.

En outre, six SEA sur 20 comportent des savoirs disciplinaires historiques et géographiques. Lorsque présente, la prise en considération des savoirs historiques ou géographiques repose surtout sur la transmission de repères historiques sur un phénomène ou sur des inventeurs célèbres (par exemple lecture d'un texte sur les technologies d'autrefois, lecture d'un texte sur la ruée vers l'or dans le Klondike, lecture d'un texte sur Copernic), sur des aspects culturels (par exemple lecture d'un texte sur les grandes peurs de l'humanité à travers le temps) ou sur des éléments du patrimoine architectural mondial (par exemple, lecture d'un texte sur les ponts et les tours célèbres dans le monde). Ces savoirs factuels renvoient aux repères culturels.

\section{Discussion}

L'ensemble des résultats démontre, d'une part, que malgré l'articulation annoncée du DGF «Environnement et consommation» aux SEA en sciences et technologies, celles-ci demeurent pour l'essentiel disciplinaires et, d'autre part, que 
l'ÉRE, lorsque présente, sollicite fort peu de savoirs historiques et géographiques. Par ailleurs, ceux qui sont sollicités restent de nature factuelle et ne servent aucunement à éclairer ou intégrer les dynamiques sociospatiales associées aux problématiques environnementales. Ils servent plutôt à contextualiser ou à mettre en perspective l'évolution de certains phénomènes. Ainsi, les rares savoirs historiques et géographiques convoqués correspondent aux repères culturels exigés par le MEQ. Il faut toutefois se garder de conclure que l'ÉRE exclut d'emblée les enjeux politiques et économiques sous-jacents aux problématiques environnementales. En fait, c'est la présence même de l'ÉRE dans les SEA analysées qu'il convient d'interroger. Le flou discursif entourant tant l'articulation du DGF aux SEA que les intentions éducatives en lien avec cette articulation laissent entrevoir une absence criante de différenciation entre le DGF «Environnement et consommation» et les apprentissages disciplinaires en sciences et technologies. Estce là le reflet d'une perspective de prolongement ou de superposition disciplinaire ou simplement le fait d'une absence de véritable prise en compte de ce domaine dans les SEA? La question demeure ouverte.

Rappelons-le, le MEQ n'évalue pas le traitement effectif des DGF dans les SEA proposées par les manuels scolaires, il se limite à en exiger l'articulation formelle. Par ailleurs, le programme de formation du primaire (MEQ, 2001) ne fournit pas aux enseignants les balises nécessaires et suffisantes devant présider à l'ancrage des DGF dans les disciplines. En outre, les axes de développement associés à ce domaine de formation intègrent des contenus qui recouvrent ceux des programmes disciplinaires et qui oscillent entre la fonction socialisante et la fonction critique. Face à une commande aussi peu précise, difficile de s'étonner du caractère diffus entourant l'articulation du domaine «Environnement et consommation» aux SEA en sciences et technologie et du peu de savoirs historiques et géographiques convoqués.

\section{Conclusion}

À défaut d'exposer explicitement les limites d'un enseignement monodisciplinaire, que ce soit celui des sciences et technologies ou de toute autre discipline pour assurer la circonscription et l'analyse des problématiques environnementales, les prescriptions curriculaires relatives à l'articulation des DGF aux disciplines risquent fort de demeurer lettre morte ou de donner lieu à une articulation largement factice et superficielle qui consiste à adjoindre ici et là quelques questions destinées à sensibiliser l'élève et à lui faire adopter des comportements jugés souhaitables, mais non débattus par les élèves du point de vue de leur pertinence individuelle et sociale. Elles risquent aussi de faire l'objet d'un traitement dans des contextes non disciplinaires, comme les campagnes de recyclage à l'école, empêchant les élèves de s'appuyer sur des savoirs homologués afin de comprendre ces problématiques et de prendre des décisions éclairées à leur sujet. Dans un cas 
comme dans l'autre, la porte est ouverte à une éducation dont l'orientation consiste à postuler que les solutions aux problématiques environnementales relèvent strictement de l'action de l'individu sans recourir nécessairement à leur conceptualisation. C'est ce qui se dégage d'une analyse de SEA de manuels de sciences et technologies destinés au premier cycle du secondaire (Hasni, 2010).

\section{Notes}

1 La notion de manuel scolaire est ici utilisée de manière générique et renvoie en même temps au manuel de l'élève et au guide de l'enseignant. De son côté, le Ministère de l'Éducation utilise la terminologie d'ensemble didactique pour désigner le manuel à l'usage de l'élève et le guide d'enseignement destiné au maître.

2 En juin 2005, le ministre de l'Éducation, du Loisir et du Sport a mis en place la Table de pilotage du renouveau pédagogique. Cette Table, formée de représentants du MELS et de divers acteurs du milieu éducatif, a pour mandat d'analyser les enjeux et obstacles en lien avec les changements curriculaires et de faire des recommandations au ministre.

\section{Références bibliographiques}

Boyatzis, R. E. (1998). Thematic analysis and code development. Transforming qualitative information. London: Sage Publications.

Commission du matériel didactique (2002). Les fonctions du matériel didactique. Avis au ministre de l'Éducation du Québec. Québec: Commission du matériel didactique.

Conseil Supérieur de l'éducation (2007). Soutenir l'appropriation des compétences transversales et des domaines généraux de formation. Avis au ministre de l'Éducation, du Loisir et du Sport. Consulté le 12 janvier 2008 dans http://www.cse.gouv.qc.ca/FR/PUBLICATIONS/index.html?l=FR

Frapet, J., Péladeau, I. \& Saint-Pierre, L. (2003). Ankor. Montréal: Modulo.

Guilbert, L. \& Gauthier, B. (1998-1999). La réflexivité en éducation environnementale: L'émergence d'une nouvelle orientation? In L. Sauvé (Éd.), Éducation relative à l'environnement: Regards, recherches, réflexions (pp. 217-223). Montréal: Université du Québec.

Hasni, A. (2010). L'éducation à l'environnement et l'interdisciplinarité: De la contextualisation des savoirs à la scolarisation du contexte? In A. Hasni \& J. Lebeaume (Éd.), Nouveaux enjeux de l'éducation scientifique et technologique: Visées, contenus, compétences et pratiques (pp. 179-222). Presses de l'Université d'Ottawa.

Hasni, A., Samson, G., Moresoli, C. \& Owen, M.-E. (2009). Points de vue d'enseignants de sciences au premier cycle du secondaire sur les manuels scolaires dans le contexte de l'implantation des nouveaux programmes au Québec. Revue des sciences de l'éducation, 35, (2), 83-105.

Landry, R. (1998). L'analyse de contenu. In B. Gauthier (Éd.), Recherche sociale. De la problématique à la collecte des données (3e éd., pp. 329-356). Presses de l'Université du Québec.

Lange, J.-M. \& Martinand, J.-L. (2010). Éducation au développement durable et éducation scientifique: Balises pour un curriculum. In A. Hasni \& J. Lebeaume (Éd.), Nouveaux enjeux de l'éducation scientifique et technologique: Visées, contenus, compétences et pratiques (pp. 125-154). Presses de l'Université d'Ottawa.

Lebrun, J., Lenoir, Y., Araújo-Oliveira, A., Morin, M.-P. \& McConnel, A.-C. (sous presse). Characteristics and contributions of school subjects at the elementary level: Results of a survey of pre-service elementary teachers from four Quebec universities. Educational Review Journal.

Létourneau, G. \& Trudeau, S. (2003). Mes chantiers. Montréal: Graficor.

Lord, F., Lytwynuk, D., Morrissette, I \& Péladeau, J. (2004). Cyclades. Montréal: Modulo. 
Luke, C., de Castell, S. \& Luke, A. (1989). Beyond criticism: The authority of the school textbook. In S. de Castell, A. Luke \& C. Luke (Éd.), Language, authority and criticism: Readings on the school textbook (pp. 245-260). London: Falmer Press.

Meirieu, P. (2001, novembre). Éduquer à l'environnement: Pourquoi? Comment? Du monde objet au monde projet. Document présenté au Congrès international Planetère, UNESCO. Résumé consulté le 10 septembre 2009 dans http://www.meirieu.com/ARTICLES/MONDE\%20OBJET_PROJET-RTF.pdf

Ministère de l'Éducation (2001). Programme de formation de l'école québécoise. Version approuvée. Éducation préscolaire. Enseignement primaire. Gouvernement du Québec.

Ministère de l'Éducation, du Loisir et du Sport (2004). Les ensembles didactiques et les critères d'évaluation. Enseignement primaire et secondaire. Gouvernement du Québec.

Sadji, H. \& Lange, J.-M., (2007, mars). Pratiques enseignantes et postures d'élèves dans le contexte de l'éducation à l'environnement pour un développement durable. Communication présentée au Colloque Les effets des pratiques enseignantes sur les apprentissages des élèves, Besançon.

Sauvé, L. (2006). Complexité et diversité du champ de l'éducation relative à l'environnement. Chemin de Traverse. Revue transdisciplinaire d'éducation à l'environnement, 3, 51-62.

Simonneaux, L. (2007, octobre). Introduction des questions scientifiques socialement vives dans l'enseignement agricole français: Implication des recherches en didactique dans la formation des enseignants. Communication présentée dans les symposiums des rencontres du Réseau Éducation Formation (REF), Sherbrooke.

Table de pilotage du renouveau pédagogique (2006). Bilan de l'application du programme de formation de l'école québécoise. Enseignement Primaire (rapport final). Québec: Ministère de l'Éducation, du Loisir et du Sport. Consulté le 18 août 2009 dans

http://www.mels.gouv.qc.ca/renouveau/pdf/TablePilotageRenouvPedFinal.pdf

Mots clés: Éducation relative à l'environnement, enseignement des sciences et technologies, manuels scolaires, savoirs historiques et géographiques, enseignement primaire

\section{Gesellschaftsblinde Umweltbildung: der Fall der Lehrmittel des Fachbereichs "Wissenschaft und Technologie» für die Primarstufe der Provinz Quebec, Kanada}

\section{Zusammenfassung}

Der vorliegende Text stellt die Analyse von 69 Lehr/Lern-Situationen aus dem Teilgebiet „Umwelt und Konsum“ des Fachbereichs „Wissenschaft und Technologie“ dar. Die untersuchten Lehr/Lern-Situationen wurden den entsprechenden Lehrmitteln für die Primarstufe der Provinz Quebec, Kanada, entnommen. Ziel der Analyse ist es, die sozial-räumlichen Dynamiken zu identifizieren, welche im Umgang mit Umweltproblemen zu berücksichtigen sind. Die Analyse weist darauf hin, dass im Fachbereich "Wissenschaft und Technologie“ trotz der dargelegten Verbindung des breiten Lernfeldes „Umwelt und Konsum“ in den Lehr/Lern-Situationen diese äusserst disziplinär gebunden bleiben und nur marginal mit Wissen über sozial-räumliche Dynamiken vernetzt werden. Dies führt dazu, dass umweltbezogene Reflexionen in hohem Masse die räumlichen, politischen und ökonomischen Dimensionen vermissen lassen. 
Schlagworte: Umweltbildung, Wissenschafts- und Technologieunterricht, Lehrmittel, geschichtliches und geographisches Wissen, Primarschulunterricht

\section{Educazione all'ambiente priva di saperi sociali: il caso dei manuali scolastici di "scienze e tecnologia" della provincia canadese del Québec}

\section{Riassunto}

L'articolo presenta un'analisi di 69 situazioni di insegnamento/apprendimento di «scienze e tecnologia» che integrano anche la formazione all'ambiente e al consumo. Queste situazioni sono contenute nei manuali scolastici per la scuola elementare. L'analisi mira a mettere in evidenza le dinamiche «socio-spaziali» che entrano in gioco quando vengono affrontati i problemi ambientali. I risultati mostrano che nonostante le questioni relative all'ambiente e al consumo siano previste nelle situazioni di apprendimento/insegnamento, il loro trattamento resta prevalentemente disciplinare e carente di riferimenti a saperi legati alle dinamiche socio-spaziali. Di conseguenza le dimensioni territoriali, politiche ed economiche sono prevalentemente neglette nelle riflessioni a carattere ambientale.

Parole chiave: Educazione ambientale, insegnamento tecnico-scientifico, manuali scolastici, insegnamento elementare

\section{Teaching Environmental Awareness without Knowledge on Society: The Case of Quebec Primary School Textbooks in Science and Technology}

\footnotetext{
Abstract

This text presents an analysis of 69 teaching/learning situations in Science and Technology that integrate the broad area of learning Consumer Habits. The examined teaching/learning situations are drawn from Quebec primary school textbooks. The analysis seeks to identify the socio-spatial dynamics taken into account when addressing environmental problems. The results show that despite the presented connection between the broad area of learning Consumer Habits and teaching/learning situations in Science and Technology, these situations remain essentially disciplinary and call upon very little knowledge relative to sociospatial dynamics. Territorial, political, and economic dimensions are therefore largely evacuated from environmental reflections.
}

Key words: Environmental education, sciences and technologies, teaching, textbooks, history and geography knowledges, primary education 